\title{
Slowly changing function connected growth properties of wronskians generated by entire and meromorphic functions
}

\author{
Sanjib Kumar Datta \\ Department of Mathematics, \\ University of Kalyani, India \\ email: sanjib_kr_datta@yahoo.co.in
}

\author{
Tanmay Biswas \\ Rajbari, Rabindrapalli, India \\ email: \\ tanmaybiswas_math@rediffmail.com
}

\author{
Ananya Kar \\ Taherpur Girls' High School, India \\ email: ananyakaronline@gmail.com
}

\begin{abstract}
In the paper we establish some new results depending on the comparative growth properties of composite entire or meromorphic functions using generalised ${ }_{p} L^{*}$-type with rate pand generalised ${ }_{p} L^{*}$-weak type with rate $p$ and wronskians generated by one of the factors.
\end{abstract}

\section{Introduction, definitions and notations}

Let $\mathbb{C}$ be the set of all finite complex numbers and $f$ be a meromorphic function defined on $\mathbb{C}$. We will not explain the standard notations and definitions in the theory of entire and meromorphic functions as those are available in [4] and [9]. In the sequel we use the following notation : $\log ^{[k]} x=\log \left(\log ^{[k-1]} x\right)$ for $k=1,2,3, \ldots$ and $\log ^{[0]} x=x$.

The following definitions are well known:

2010 Mathematics Subject Classification: 30D35,30D30

Key words and phrases: transcendental entire function, transcendental meromorphic function, composition, growth, generalised $\mathrm{p}^{*}$-type with rate $p$ and generalised $\mathrm{L}^{*}$-weak type with rate $p$, wronskian, slowly changing function 
Definition 1 A meromorphic function $\mathrm{a} \equiv \mathrm{a}(\mathrm{z})$ is called small with respect to $f$ if $\mathrm{T}(\mathrm{r}, \mathrm{a})=\mathrm{S}(\mathrm{r}, \mathrm{f})$.

Definition 2 Let $\mathrm{a}_{1}, \mathrm{a}_{2}, \ldots . \mathrm{a}_{\mathrm{k}}$ be linearly independent meromorphic functions and small with respect to $f$. We denote by $L(f)=W\left(a_{1}, a_{2}, \ldots . a_{k} ; f\right)$ the Wronskian determinant of $\mathrm{a}_{1}, \mathrm{a}_{2}, \ldots ., \mathrm{a}_{\mathrm{k}}$, f i.e.,

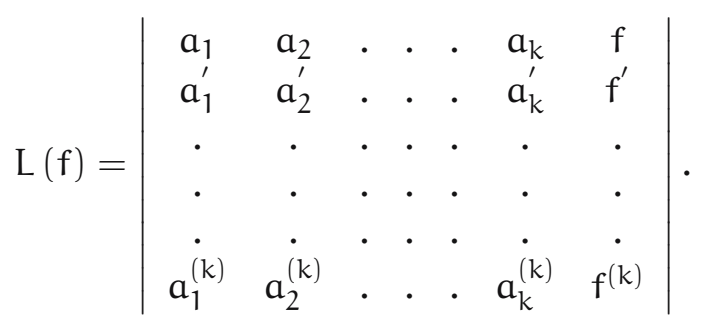

Definition 3 If $\mathrm{a} \in \mathbb{C} \cup\{\infty\}$, the quantity

$$
\begin{aligned}
\delta(a ; f) & =1-\limsup _{r \rightarrow \infty} \frac{N(r, a ; f)}{T(r, f)} \\
& =\liminf _{r \rightarrow \infty} \frac{m(r, a ; f)}{T(r, f)}
\end{aligned}
$$

is called the Nevanlinna deficiency of the value ' $\mathrm{a}$ '.

From the second fundamental theorem it follows that the set of values of $a \in \mathbb{C} \cup\{\infty\}$ for which $\delta(a ; f)>0$ is countable and $\sum_{a \neq \infty} \delta(a ; f)+\delta(\infty ; f) \leq 2$ (cf [4], p. 43). If in particular, $\sum_{a \neq \infty} \delta(a ; f)+\delta(\infty ; f)=2$, we say that $f$ has the maximum deficiency sum.

Let $\mathrm{L} \equiv \mathrm{L}(\mathrm{r}$ ) be a positive continuous function increasing slowly i.e., $\mathrm{L}$ (ar) $\mathrm{L}(\mathrm{r})$ as $r \rightarrow \infty$ for every positive constant a. Singh and Barker [7] defined it in the following way:

Definition 4 [7] A positive continuous function $\mathrm{L}(\mathrm{r})$ is called a slowly changing function if for $\varepsilon(>0)$,

$$
\frac{1}{\mathrm{k}^{\varepsilon}} \leq \frac{\mathrm{L}(\mathrm{kr})}{\mathrm{L}(\mathrm{r})} \leq \mathrm{k}^{\varepsilon} \text { for } \mathrm{r} \geq \mathrm{r}(\varepsilon) \text { and }
$$

uniformly for $\mathrm{k}(\geq 1)$. 
Somasundaram and Thamizharasi [8] introduced the notions of L-order and L-lower order for entire function where $\mathrm{L} \equiv \mathrm{L}(\mathrm{r})$ is a positive continuous function increasing slowly i.e., L (ar) $\sim \mathrm{L}(\mathrm{r})$ as $r \rightarrow \infty$ for every positive constant ' $a$ '. The more generalized concept for L-order and L-lower order for entire function are $L^{*}$-order and $L^{*}$-lower order. Their definitions are as follows:

Definition 5 [8] The $\mathrm{L}^{*}$-order $\rho_{\mathrm{f}}^{\mathrm{L}^{*}}$ and the $\mathrm{L}^{*}$-lower order $\lambda_{f}^{\mathrm{L}^{*}}$ of an entire function $\mathrm{f}$ are defined as

$$
\rho_{f}^{\mathrm{L}^{*}}=\limsup _{r \rightarrow \infty} \frac{\log ^{[2]} M(r, f)}{\log \left[r e^{\mathrm{L}(r)}\right]} \text { and } \lambda_{f}^{\mathrm{L}^{*}}=\liminf _{r \rightarrow \infty} \frac{\log ^{[2]} M(r, f)}{\log \left[r e^{\mathrm{L}(r)}\right]} \text {. }
$$

When $\mathrm{f}$ is meromorphic, the above definition reduces to

$$
\rho_{f}^{\mathrm{L}^{*}}=\limsup _{r \rightarrow \infty} \frac{\log \mathrm{T}(\mathrm{r}, \mathrm{f})}{\log \left[r e^{\mathrm{L}(\mathrm{r})}\right]} \text { and } \lambda_{f}^{\mathrm{L}^{*}}=\liminf _{r \rightarrow \infty} \frac{\log \mathrm{T}(\mathrm{r}, \mathrm{f})}{\log \left[r e^{\mathrm{L}(\mathrm{r})}\right]} .
$$

In the line of Somasundaram and Thamizharasi [8], for any two positive integers $m$ and $p$, Datta and Biswas [1] introduced the following definition:

Definition 6 [1] The $\mathrm{m}$-th generalized $\mathrm{p}^{*}$-order with rate $\mathrm{p}$ denoted by $(\mathrm{p}) \rho_{\mathrm{f}}^{(\mathrm{m})} \rho^{\mathrm{L}^{*}}$ and the $\mathrm{m}$-th generalized $\mathrm{p}^{\mathrm{L}^{*}}$-lower order with rate $\mathrm{p}$ denoted as $\underset{(\mathrm{p})}{(\mathrm{m})} \lambda_{\mathrm{f}}^{\mathrm{L}^{*}}$ of an entire function $\mathrm{f}$ are defined in the following way:

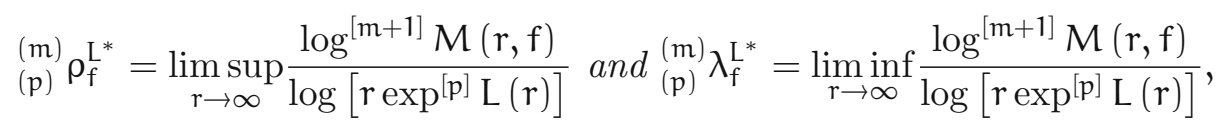

where both $\mathrm{m}$ and $\mathrm{p}$ are positive integers.

When $\mathrm{f}$ is meromorphic, it can be easily verified that

$$
\underset{(\mathrm{p})}{(\mathrm{m})} \rho_{f}^{\mathrm{L}^{*}}=\limsup _{\mathrm{r} \rightarrow \infty} \frac{\log ^{[\mathrm{m}]} \mathrm{T}(\mathrm{r}, \mathrm{f})}{\log \left[\mathrm{r} \exp ^{[\mathrm{p}]} \mathrm{L}(\mathrm{r})\right]} \text { and } \underset{(\mathrm{p})}{(\mathrm{m})} \lambda_{f}^{\mathrm{L}^{*}}=\liminf _{\mathrm{r} \rightarrow \infty} \frac{\log ^{[\mathrm{m}]} \mathrm{T}(\mathrm{r}, \mathrm{f})}{\log \left[\mathrm{r} \exp ^{[\mathrm{p}]} \mathrm{L}(\mathrm{r})\right]} \text {, }
$$

where both $\mathrm{m}$ and $\mathrm{p}$ are positive integers.

To compare the relative growth of two entire or meromorphic functions having same non zero finite generalized $\mathrm{L}^{*}$-order with rate $p$, one may introduce the definitions of generalised ${ }_{p} L^{*}$-type with rate $p$ and generalised ${ }_{p} L^{*}$-lower type with rate $p$ of entire and meromorphic functions having finite positive generalised ${ }_{p} L^{*}$-order with rate $p$ in the following manner: 
Definition 7 The $\mathrm{m}$-th generalised $\mathrm{p}^{*}$-type with rate $\mathrm{p}$ denoted by $y_{(\mathrm{p})}^{(\mathrm{m})} \sigma_{\mathrm{f}}^{\mathrm{L}^{*}}$ and $\mathrm{m}$-th generalised $\mathrm{p}^{*}$-lower type with rate $\mathrm{p}$ of an entire function $\mathrm{f}$ denoted by ${ }_{(\mathrm{p})}^{(\mathrm{m})} \bar{\sigma}_{\mathrm{f}}^{\mathrm{L}^{*}}$ are respectively defined as follows:

$$
\begin{aligned}
& \underset{(\mathrm{p})}{(\mathrm{m})} \sigma_{f}^{\mathrm{L}^{*}}=\limsup _{\mathrm{r} \rightarrow \infty} \frac{\log ^{[\mathrm{m}]} \mathrm{M}(\mathrm{r}, \mathrm{f})}{\left[r \exp ^{[\mathrm{p}]} \mathrm{L}(\mathrm{r})\right]^{(\mathrm{m})} \rho_{\mathrm{f}}^{\mathrm{L}^{*}}} \text { and } \\
& \underset{(p)}{(m)} \bar{\sigma}_{f}^{L^{*}}=\liminf _{r \rightarrow \infty} \frac{\log ^{[m]} M(r, f)}{\left[r \exp ^{[p]} L(r)\right]^{(m)} \rho_{f}^{L^{*}}}, 0<\underset{(p)}{(m)} \rho_{f}^{L^{*}}<\infty,
\end{aligned}
$$

where $\mathrm{m}$ and $\mathrm{p}$ are any two positive integers.

For meromorphic f,

$$
\begin{aligned}
& \underset{(\mathrm{p})}{(\mathrm{m})} \sigma_{\mathrm{f}}^{\mathrm{L}^{*}}=\limsup _{\mathrm{r} \rightarrow \infty} \frac{\log ^{[\mathrm{m}-1]} \mathrm{T}(\mathrm{r}, \mathrm{f})}{\left[\mathrm{r} \exp ^{[\mathrm{p}]} \mathrm{L}(\mathrm{r})\right]^{(\mathrm{m})} \rho_{\mathrm{f}}^{\mathrm{L}^{*}}} \text { and } \\
& { }_{(\mathfrak{p})}^{(m)} \bar{\sigma}_{f}^{L^{*}}=\liminf _{r \rightarrow \infty} \frac{\log ^{[m-1]} \mathrm{T}(r, f)}{\left[r \exp ^{[p]} \mathrm{L}(r)\right]^{(m)} \rho_{f}^{L^{*}}}, 0<{ }_{(p)}^{(m)} \rho_{f}^{L^{*}}<\infty,
\end{aligned}
$$

where both $\mathrm{m}$ and $\mathrm{p}$ are positive integers.

Analogously to determine the relative growth of two entire or meromorphic functions having same non zero finite generalized ${ }_{p} L^{*}$-lower order with rate $p$ one may introduce the definition of generalised ${ }_{p} L^{*}$-weak type with rate $p$ of entire and meromorphic functions having finite positive generalized ${ }_{p} \mathrm{~L}^{*}$-lower order with rate $p$ in the following way:

Definition 8 The $\mathrm{m}$-th generalised $\mathrm{p}{ }^{*}$-weak type with rate $\mathrm{p}$ denoted by ${ }_{(\mathrm{p})}^{(\mathrm{m})} \tau_{\mathrm{f}}^{\mathrm{L}^{*}}$ of an entire function $\mathrm{f}$ is defined as follows:

$$
\underset{(p)}{(m)} \tau_{f}^{L^{*}}=\liminf _{r \rightarrow \infty} \frac{\log ^{[m]} M(r, f)}{\left[r \exp ^{[p]} L(r)\right]^{(m)} \lambda_{f}^{L^{*}}}, 0<{ }_{(p)}^{(m)} \lambda_{f}^{L^{*}}<\infty,
$$

where both $\mathrm{m}$ and $\mathrm{p}$ are positive integers.

Also one may define the growth indicator ${ }_{(\mathrm{p})}^{(\mathrm{m})} \bar{\tau}_{\mathrm{f}}^{\mathrm{L}^{*}}$ of an entire function $\mathrm{f}$ in the following manner:

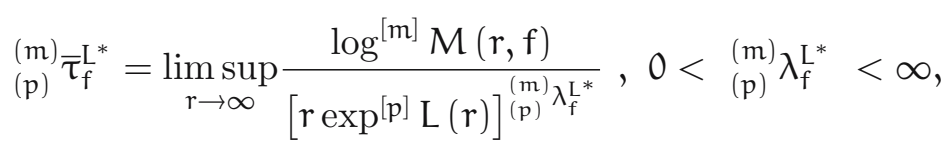


where $\mathrm{m}$ and $\mathrm{p}$ are any two positive integers.

For meromorphic f,

$$
\begin{aligned}
& \underset{(\mathrm{p})}{(\mathrm{m})} \bar{\tau}_{\mathrm{f}}^{\mathrm{L}^{*}}=\limsup _{\mathrm{r} \rightarrow \infty} \frac{\log ^{[\mathrm{m}-1]} \mathrm{T}(\mathrm{r}, \mathrm{f})}{\left[\mathrm{r} \exp ^{[\mathrm{p}]} \mathrm{L}(\mathrm{r})\right]^{(\mathrm{m}))^{(\mathrm{m})} \lambda_{\mathrm{f}}^{\mathrm{L}^{*}}}} \text { and } \\
& \underset{(\mathrm{p})}{(\mathrm{m})} \tau_{\mathrm{f}}^{\mathrm{L}^{*}}=\liminf _{\mathrm{r} \rightarrow \infty} \frac{\log ^{[\mathrm{m}-1]} \mathrm{T}(\mathrm{r}, \mathrm{f})}{\left[\mathrm{r} \exp ^{[p]} \mathrm{L}(\mathrm{r})\right]^{(\mathrm{m})} \lambda_{\mathrm{f}}^{\mathrm{L}^{*}}}, 0<\underset{(\mathrm{p})}{(\mathrm{m})} \lambda_{f}^{\mathrm{L}^{*}}<\infty,
\end{aligned}
$$

where both $\mathrm{m}$ and $\mathrm{p}$ are positive integers.

Lakshminarasimhan [5] introduced the idea of the functions of L-bounded index. Later Lahiri and Bhattacharjee [6] worked on the entire functions of L-bounded index and of non uniform L-bounded index. Since the natural extension of a derivative is a differential polynomial, in this paper we prove our results for a special type of linear differential polynomials viz. the Wronskians. In the paper we establish some new results depending on the comparative growth properties of composite entire or meromorphic functions using generalised $L^{*}$-order with rate $p$, generalised $L^{*}$ - type with rate $p$ and generalised $p L^{*}$-weak type with rate $p$ and wronskians generated by one of the factors which extend some results of [2].

\section{Lemmas}

In this section we present some lemmas which will be needed in the sequel.

Lemma 1 [3] Let $\mathrm{f}$ be a transcendental meromorphic function having the maximum deficiency sum. Then

(i) $(p)^{(m)} \sigma_{L(f)}^{L^{*}}=\{1+k-k \delta(\infty ; f)\} \cdot(p)^{(m)} \sigma_{f}^{L^{*}}$ for $m=1$ and

$(p)^{(m)} \sigma_{L(f)}^{L^{*}}={ }_{(p)}^{(m)} \sigma_{f}^{L^{*}}$ otherwise

and

(ii) $(p)^{(m)} \bar{\sigma}_{L(f)}^{L^{*}}=\{1+k-k \delta(\infty ; f)\} \cdot(p)^{(m)} \bar{\sigma}_{f}^{L^{*}}$ for $m=1$ and $(p)^{(m)} \bar{\sigma}_{L(f)}^{L^{*}}={ }_{(p)}^{(m)} \bar{\sigma}_{f}^{L^{*}}$ otherwise. 
Lemma 2 [3] Let $\mathrm{f}$ be a transcendental meromorphic function having the maximum deficiency sum. Then

$$
\begin{aligned}
(i)(p)^{(m)} \tau_{L(f)}^{L^{*}} & =\{1+k-k \delta(\infty ; f)\} \cdot(p)^{(m)} \tau_{f}^{L^{*}} \text { for } m=1 \text { and } \\
(p)^{(m)} \tau_{L(f)}^{L^{*}} & ={ }_{(p)}^{(m)} \tau_{f}^{L^{*}} \text { otherwise }
\end{aligned}
$$

and

$$
\begin{aligned}
& \text { (ii) }(p)^{(m)} \bar{\tau}_{L(f)}^{L^{*}}=\{1+k-k \delta(\infty ; f)\} \cdot{ }_{(p)}^{(m)} \bar{\tau}_{f}^{L^{*}} \text { for } m=1 \text { and } \\
& (p)^{(m)} \bar{\tau}_{L(f)}^{L^{*}}={ }_{(p)}^{(m)} \bar{\tau}_{f}^{L^{*}} \text { otherwise. }
\end{aligned}
$$

\section{Theorems}

In this section we present the main results of the paper.

Theorem 1 If $\mathrm{f}$ be transcendental meromorphic and $\mathrm{g}$ be entire such that $0<{ }_{(p)}^{(m)} \bar{\sigma}_{f \circ g}^{L^{*}} \leq{ }_{(p)}^{(m)} \sigma_{f \circ g}^{L^{*}}<\infty, 0<\underset{(p)}{(n)} \bar{\sigma}_{f}^{L^{*}} \leq{ }_{(p)}^{(n)} \sigma_{f}^{L^{*}}<\infty, \underset{(p)}{(m)} \rho_{f \circ g}^{L^{*}}={ }_{(p)}^{(n)} \rho_{f}^{L^{*}}$ and $\sum_{a \neq \infty} \delta(a ; f)+\delta(\infty ; f)=2$ where $m, n$ and $p$ are any three positive integers, then

$$
\begin{aligned}
& \frac{{ }_{(\mathfrak{m})}^{(m)} \bar{\sigma}_{f \circ g}^{L^{*}}}{\{1+k-k \delta(\infty ; f)\} \cdot(p) \sigma_{f}^{L^{*}}} \leq \liminf _{r \rightarrow \infty} \frac{\log ^{[m-1]} \mathrm{T}(r, f \circ g)}{T(r, L(f))} \\
& \leq \frac{(\mathrm{m})}{\mathrm{\sigma}_{\mathrm{f} \circ \mathrm{g}}^{\mathrm{L}^{*}}} \frac{(\mathrm{p}) \bar{\sigma}_{\mathrm{f}}^{\mathrm{L}^{*}}}{\{1+\mathrm{k}-\mathrm{k} \delta(\infty)\} \cdot(\mathrm{f})} \\
& \leq \limsup _{r \rightarrow \infty} \frac{\log ^{[m-1]} \mathrm{T}(\mathrm{r}, \mathrm{f} \circ \mathrm{g})}{\mathrm{T}(\mathrm{r}, \mathrm{L}(\mathrm{f}))}
\end{aligned}
$$

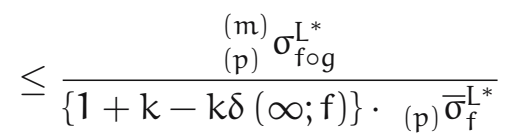

and

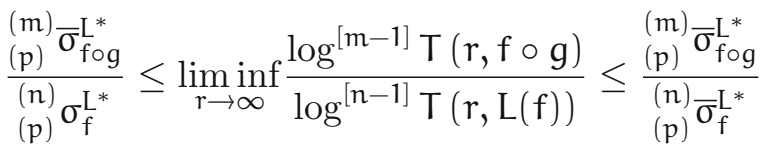

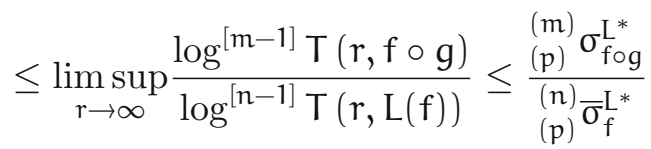

for $\mathrm{n}>1$. 
Proof. From the definition of $\underset{(p)}{(n)} \sigma_{L(f)}^{L^{*}}, \frac{(m)}{(p)} \bar{\sigma}_{f \circ g}^{L^{*}}$ and in view of Lemma 1, we have for arbitrary positive $\varepsilon$ and for all sufficiently large values of $r$ that

$$
\begin{aligned}
& \log ^{[m-1]} \mathrm{T}(r, f \circ g) \geq\left(\begin{array}{l}
(m) \\
(p)
\end{array} \bar{\sigma}_{f \circ g}^{L^{*}}-\varepsilon\right)\left[r \exp ^{[p]} L(r)\right]^{(\mathfrak{p}) \rho_{f \circ g}^{L^{*}}},
\end{aligned}
$$

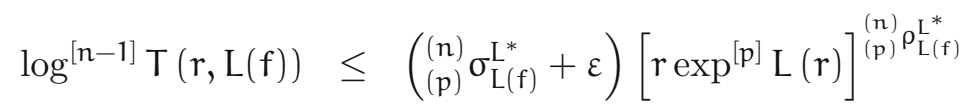

$$
\begin{aligned}
& \text { i.e., } \log ^{[n-1]} T(r, L(f)) \leq\left(\begin{array}{l}
(\mathfrak{n}) \\
(\mathfrak{p})
\end{array} \sigma_{f}^{L^{*}}+\varepsilon\right)\left[r \exp ^{[p]} L(r)\right]^{(\mathfrak{n})} \rho^{L_{f}^{*}}
\end{aligned}
$$

for $n>1$ and

$$
\mathrm{T}(\mathrm{r}, \mathrm{L}(\mathrm{f})) \leq\{1+\mathrm{k}-\mathrm{k} \delta(\infty ; f)\} \cdot\left({ }_{(p)} \sigma_{f}^{\mathrm{L}^{*}}+\varepsilon\right)\left[r \exp ^{[p]} \mathrm{L}(\mathrm{r})\right]^{(p) \rho_{\mathrm{f}}^{\mathrm{L}^{*}}} .
$$

Now from $(1),(2)$ and the condition $\underset{(\mathfrak{p})}{(\mathrm{m})} \rho_{\mathrm{f} \circ \mathrm{g}}^{\mathrm{L}^{*}}=\underset{(\mathrm{p})}{(\mathfrak{n})} \rho_{\mathrm{f}}^{\mathrm{L}}$, it follows for all sufficiently large values of $r$ that,

$$
\frac{\log ^{[m-1]} T(r, f \circ g)}{\log ^{[n-1]} T(r, L(f))} \geqslant \frac{(\mathfrak{m}) \bar{\sigma}_{\text {fog }}^{L^{*}}-\varepsilon}{(\mathfrak{n}) \sigma_{f}^{L^{*}}+\varepsilon} \text { for } n>1 .
$$

As $\varepsilon(>0)$ is arbitrary, we obtain from above that

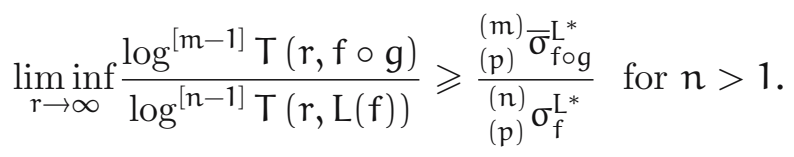

Similarly from $(1),(3)$ and in view of the condition $\underset{(\mathfrak{p})}{(\mathfrak{m})} \rho_{\text {fog }}^{\mathrm{L}^{*}}={ }_{(\mathrm{p})} \rho_{\mathrm{f}}^{\mathrm{L}}$, we obtain that

$$
\liminf _{r \rightarrow \infty} \frac{\log ^{[m-1]} T(r, f \circ g)}{T(r, L(f))} \geqslant \frac{(m)}{\{1+k-k \delta(\infty ; f)\} \cdot{ }_{(p)}^{L_{f}^{*}} \sigma_{f}^{L^{*}}} .
$$

Again for a sequence of values of $r$ tending to infinity,

$$
\log ^{[m-1]} T(r, f \circ g) \leq\left(\begin{array}{l}
(m) \\
(p)
\end{array} \bar{\sigma}_{f \circ g}^{L^{*}}+\varepsilon\right)\left[r \exp ^{[p]} L(r)\right]^{(\mathfrak{m})} \rho_{f \circ g}^{L_{f *}^{*}}
$$

and for all sufficiently large values of $r$,

$$
\begin{aligned}
& \log ^{[n-1]} T(r, L(f)) \geq\left(\begin{array}{l}
(\mathfrak{n}) \\
(\mathfrak{p})
\end{array} \bar{\sigma}_{\mathrm{L}(f)}^{\mathrm{L}^{*}}-\varepsilon\right)\left[r \exp ^{[p]} \mathrm{L}(\mathrm{r})\right]^{(\mathfrak{n}))^{(\mathrm{n})} \mathrm{L}^{*}(f)}
\end{aligned}
$$

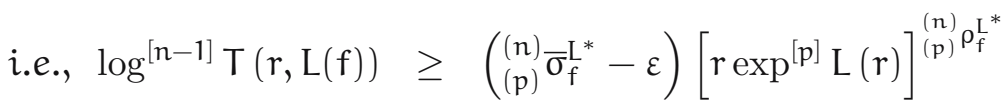


for $n>1$ and

$$
\mathrm{T}(\mathrm{r}, \mathrm{L}(\mathrm{f})) \geq\{1+\mathrm{k}-\mathrm{k} \delta(\infty ; f)\} \cdot\left({ }_{(\mathfrak{p})} \bar{\sigma}_{f}^{\mathrm{L}^{*}}-\varepsilon\right)\left[\mathrm{r} \exp ^{[\mathrm{p}]} \mathrm{L}(\mathrm{r})\right]^{(\mathfrak{p}) \rho_{\mathrm{f}}^{\mathrm{L}^{*}}} .
$$

Combining (6) and (7) and the condition $\underset{(p)}{(m)} \rho_{f \circ g}^{L^{*}}={ }_{(p)}^{(n)} \rho_{f}^{L}$, we get for a sequence of values of $r$ tending to infinity that

$$
\frac{\log ^{[m-1]} T(r, f \circ g)}{\log ^{[n-1]} T(r, L(f))} \leq \frac{(\mathfrak{p}) \bar{\sigma}_{f \circ g}^{L^{*}}+\varepsilon}{(\mathfrak{n}) \bar{\sigma}_{f}^{L^{*}}-\varepsilon} \text { for } n>1 .
$$

Since $\varepsilon(>0)$ is arbitrary, it follows from above that

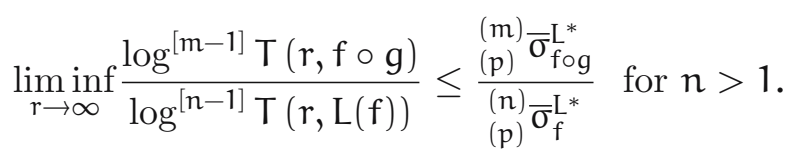

Likewise from (6) and (8) and in view of the condition $\underset{(\mathrm{p})}{(\mathrm{m})} \rho_{\mathrm{fog}}^{\mathrm{L}^{*}}={ }_{(\mathrm{p})} \rho_{\mathrm{f}}^{\mathrm{L}}$, we obtain that

$$
\liminf _{r \rightarrow \infty} \frac{\log ^{[m-1]} T(r, f \circ g)}{T(r, L(f))} \leq \frac{(m)}{\{1+k-k \delta(\infty ; f)\} \cdot(p) \bar{\sigma}_{f}^{L^{*}}} .
$$

Also for a sequence of values of $r$ tending to infinity it follows that

$$
\begin{aligned}
& \left.\log ^{[n-1]} \mathrm{T}(\mathrm{r}, \mathrm{L}(\mathrm{f})) \leq\left(\begin{array}{l}
(\mathfrak{n}) \\
(\mathfrak{p})
\end{array} \bar{\sigma}_{\mathrm{L}(\mathrm{f})}^{\mathrm{L}^{*}}\right\}+\varepsilon\right)\left[\mathrm{r} \exp ^{[\mathrm{p}]} \mathrm{L}(\mathrm{r})\right]^{(\mathfrak{n})} \rho^{\mathrm{L}_{\mathrm{L}(f)}}
\end{aligned}
$$

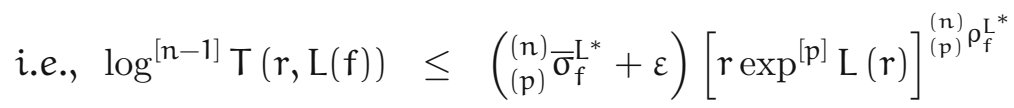

for $n>1$ and

$$
\mathrm{T}(\mathrm{r}, \mathrm{L}(\mathrm{f})) \leq\{1+\mathrm{k}-\mathrm{k} \delta(\infty ; f)\} \cdot\left({ }_{(\mathrm{p})} \bar{\sigma}_{\mathrm{f}}^{\mathrm{L}^{*}}+\varepsilon\right)\left[\mathrm{r} \exp ^{[\mathrm{p}]} \mathrm{L}(\mathrm{r})\right]^{(\mathrm{p}) \rho_{\mathrm{f}}^{\mathrm{L}^{*}}} .
$$

Now from $(1),(11)$ and the condition $\underset{(p)}{(m)} \rho_{f \circ g}^{L^{*}}=\underset{(p)}{(\mathfrak{n})} \rho_{f}^{\mathrm{L}}$, we obtain for a sequence of values of $r$ tending to infinity that

$$
\frac{\log ^{[m-1]} T(r, f \circ g)}{\log ^{[n-1]} T(r, L(f))} \geq \frac{(\mathfrak{p}) \bar{\sigma}_{f \circ g}^{L^{*}}-\varepsilon}{(\mathfrak{n}) \bar{\sigma}_{f}^{L^{*}}+\varepsilon} \text { for } n>1
$$


As $\varepsilon(>0)$ is arbitrary, we get from above that

$$
\limsup _{r \rightarrow \infty} \frac{\log ^{[m-1]} T(r, f \circ g)}{\log ^{[n-1]} T(r, L(f))} \geq \frac{(m)}{(\mathfrak{m}) \bar{\sigma}_{f \circ g}^{L^{*}}} \text { for } n>1 .
$$

Analogously from (1), (12) and in view of the condition $\underset{(\mathrm{p})}{(\mathrm{m})} \rho_{\mathrm{f} \circ \mathrm{g}}^{\mathrm{L}^{*}}={ }_{(\mathrm{p})} \rho_{\mathrm{f}}^{\mathrm{L}}$, we get that

$$
\limsup _{r \rightarrow \infty} \frac{\log ^{[m-1]} T(r, f \circ g)}{T(r, L(f))} \geq \frac{(\mathfrak{m}) \bar{\sigma}_{f \circ g}^{L^{*}}}{\{1+k-k \delta(\infty ; f)\} \cdot(p) \bar{\sigma}_{f}^{L^{*}}} .
$$

Also for all sufficiently large values of $r$,

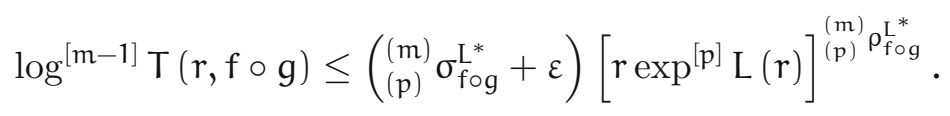

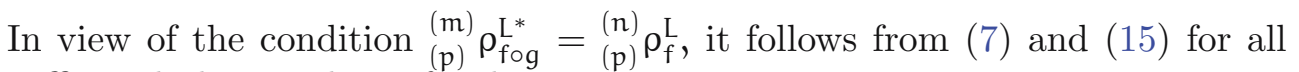
sufficiently large values of $r$ that

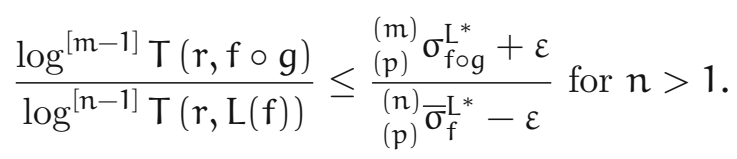

Since $\varepsilon(>0)$ is arbitrary, we obtain that

$$
\limsup _{r \rightarrow \infty} \frac{\log ^{[m-1]} T(r, f \circ g)}{\log ^{[n-1]} T(r, L(f))} \leq \frac{(m)}{(\mathfrak{p}) \sigma_{f \circ g}^{L^{*}}} \text { for } n>1
$$

Similarly from (8) and (15) and in view of the condition $\underset{(\mathfrak{p})}{(\mathrm{m})} \rho_{\mathrm{fog}}^{\mathrm{L}^{*}}={ }_{(\mathrm{p})} \rho_{\mathrm{f}}^{\mathrm{L}}$, we obtain that

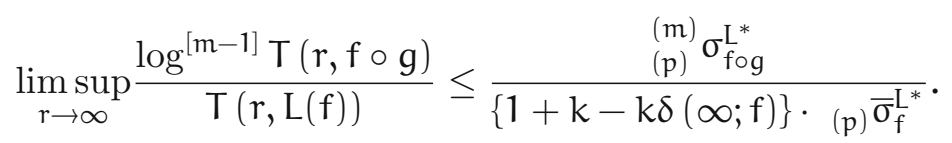

Thus the theorem follows from (4), (5), (9), (10), (13), (14), (16) and (17).

The following theorem can be proved in the line of Theorem 1 and so its proof is omitted. 
Theorem 2 If $\mathrm{f}$ be meromorphic and $\mathrm{g}$ be transcendental entire with $0<$ ${ }_{(\mathrm{p})}^{(\mathrm{m})} \bar{\sigma}_{f \circ g}^{\mathrm{L}^{*}} \leq{ }_{(\mathrm{p})}^{(\mathrm{m})} \sigma_{f \circ g}^{\mathrm{L}^{*}}<\infty, 0<{ }_{(\mathrm{p})}^{(\mathrm{n})} \bar{\sigma}_{g}^{\mathrm{L}^{*}} \leq{ }_{(\mathrm{p})}^{(\mathrm{n})} \sigma_{\mathrm{g}}^{\mathrm{L}^{*}}<\infty,{ }_{(\mathrm{p})}^{(\mathrm{m})} \rho_{f \circ g}^{\mathrm{L}^{*}}={ }_{(\mathrm{p})}^{(\mathrm{n})} \rho_{g}^{\mathrm{L}^{*}}$ and $\sum_{a \neq \infty} \delta(a ; g)+\delta(\infty ; g)=2$ where $m, n$ and $p$ are any three positive integers, then

$$
\begin{aligned}
\frac{(m)}{(p)} \bar{\sigma}_{f \circ g}^{L^{*}} & \leq \liminf _{r \rightarrow \infty} \frac{\log ^{[m-1]} T(r, f \circ g)}{T(r, L(g))} \\
& \leq \frac{(p) \sigma_{f \circ g}}{\{1+k-k \delta(\infty ; g)\} \cdot{ }_{(p)} \sigma_{g}^{L^{*}}} \\
& \leq \limsup _{r \rightarrow \infty} \frac{\log ^{[m-1]} T(r, f \circ g)}{T(r, L(g))} \\
& \leq \frac{(m)}{\{1+k-k \delta(\infty ; g)\} \cdot(p) \bar{\sigma}_{g}^{L^{*}}}
\end{aligned}
$$

and

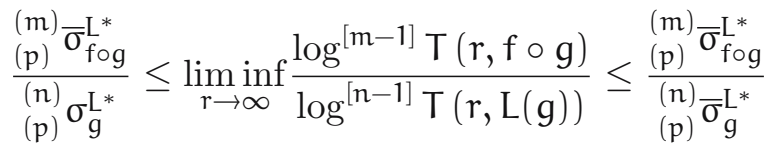

$$
\begin{aligned}
& \leq \limsup _{r \rightarrow \infty} \frac{\log ^{[m-1]} \mathrm{T}(r, f \circ g)}{\log ^{[n-1]} \mathrm{T}(\mathrm{r}, \mathrm{L}(\mathrm{g}))} \leq \frac{(\mathrm{m})}{(\mathrm{p})} \sigma_{\mathrm{f} \circ \mathrm{g}}^{\mathrm{L}^{*}}
\end{aligned}
$$

for $\mathrm{n}>1$.

Theorem 3 If $\mathrm{f}$ be transcendental meromorphic and $\mathrm{g}$ be entire such that $0<$ $\underset{(p)}{(m)} \sigma_{f \circ g}^{L^{*}}<\infty, 0<\underset{(p)}{(n)} \sigma_{f}^{L^{*}}<\infty, \underset{(p)}{(m)} \rho_{f \circ g}^{L^{*}}=\underset{(p)}{(n)} \rho_{f}^{L^{*}}$ and $\sum_{a \neq \infty} \delta(a ; f)+\delta(\infty ; f)=2$ where $\mathrm{m}, \mathrm{n}$ and $\mathrm{p}$ are any three positive integers, then

$$
\begin{aligned}
\liminf _{r \rightarrow \infty} \frac{\log ^{[m-1]} T(r, f \circ g)}{T(r, L(f))} & \leq \frac{(p)}{\{1+k-k \delta(\infty ; f)\} \cdot{ }_{f(p)} \sigma_{f}^{L^{*}}} \\
& \leq \limsup _{r \rightarrow \infty} \liminf _{r \rightarrow \infty} \frac{\log ^{[m-1]} T(r, f \circ g)}{T(r, L(f))}
\end{aligned}
$$

and

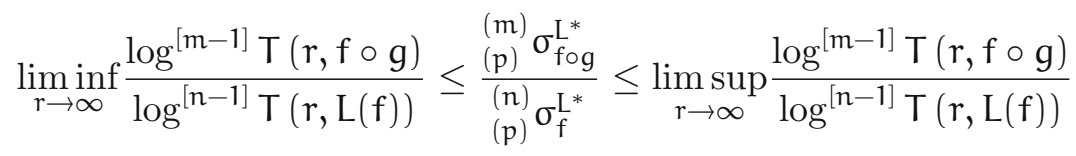


for $\mathrm{n}>1$.

Proof. From the definition of $\underset{(p)}{(n)} \sigma_{L(f)}^{L^{*}}$ and in view of Lemma 1, we get for a sequence of values of $r$ tending to infinity that

$$
\begin{aligned}
& \left.\log ^{[n-1]} \mathrm{T}(\mathrm{r}, \mathrm{L}(\mathrm{f})) \geq\left(\begin{array}{l}
(\mathfrak{n}) \\
(\mathfrak{p})
\end{array} \sigma_{\mathrm{L}(\mathrm{f})}^{\mathrm{L}^{*}}\right\}-\varepsilon\right)\left[r \exp ^{[\mathrm{p}]} \mathrm{L}(\mathrm{r})\right]^{(\mathfrak{n})} \rho^{\rho_{\mathrm{L}(f)}^{\mathrm{L}^{*}}} \\
& \text { i.e., } \log ^{[\mathfrak{n}-1]} \mathrm{T}(\mathrm{r}, \mathrm{L}(\mathrm{f})) \geq\left(\begin{array}{l}
(\mathfrak{n}) \\
(\mathrm{p})
\end{array} \sigma_{f}^{L^{*}}-\varepsilon\right)\left[r \exp ^{[\mathrm{p}]} \mathrm{L}(\mathrm{r})\right]^{(\mathfrak{p})} \rho^{(\mathfrak{n})}
\end{aligned}
$$

for $n>1$ and

$$
\mathrm{T}(\mathrm{r}, \mathrm{L}(\mathrm{f})) \geq\{1+k-k \delta(\infty ; f)\} \cdot\left({ }_{(\mathrm{p})} \sigma_{f}^{\mathrm{L}^{*}}-\varepsilon\right)\left[r \exp ^{[\mathrm{p}]} \mathrm{L}(\mathrm{r})\right]^{(\mathrm{p}))_{\mathrm{f}}^{\mathrm{L}^{*}}} .
$$

Now from $(15),(18)$ and the condition $\underset{(\mathfrak{p})}{(m)} \rho_{f \circ g}^{\mathrm{L}^{*}}=\underset{(p)}{(\mathfrak{n})} \rho_{\mathrm{f}}^{\mathrm{L}^{*}}$, it follows for a sequence of values of $r$ tending to infinity that

$$
\frac{\log ^{[m-1]} T(r, f \circ g)}{\log ^{[n-1]} T(r, L(f))} \leq \frac{(m)}{(\mathfrak{p}) \sigma_{f \circ g}^{L^{*}}+\varepsilon} \text { for } n>1 .
$$

As $\varepsilon(>0)$ is arbitrary, we obtain that

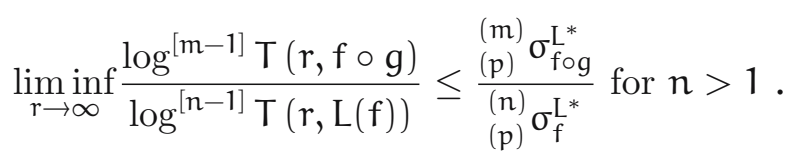

Similarly from $(15),(19)$ and in view of the condition $\underset{(\mathfrak{p})}{(\mathrm{m})} \rho_{\mathrm{fog}}^{\mathrm{L}^{*}}={ }_{(\mathfrak{p})} \rho_{\mathrm{f}}^{\mathrm{L}}$, we obtain that

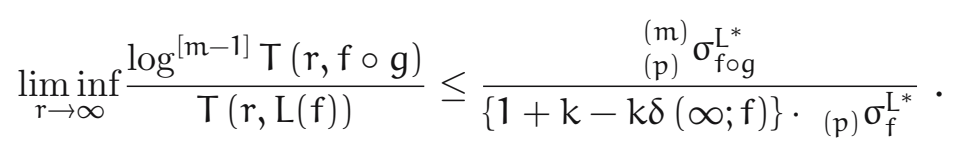

Again for a sequence of values of $r$ tending to infinity that

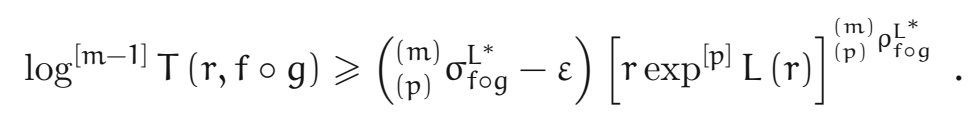

So combining (2) and (22) and in view of the condition $\underset{(\mathfrak{p})}{(\mathfrak{m})} \rho_{f \circ g}^{\mathrm{L}^{*}}=\underset{(\mathfrak{p})}{(\mathfrak{n})} \rho_{f}^{\mathrm{L}^{*}}$, we get for a sequence of values of $r$ tending to infinity that

$$
\frac{\log ^{[m-1]} T(r, f \circ g)}{\log ^{[n-1]} T(r, L(f))} \geqslant \frac{(m)}{(\mathfrak{p}) \sigma_{\text {fog }}^{L^{*}}-\varepsilon} \text { for } n>1 .
$$


Since $\varepsilon(>0)$ is arbitrary, it follows that

$$
\limsup _{r \rightarrow \infty} \frac{\log ^{[m-1]} T(r, f \circ g)}{\log ^{[n-1]} T(r, L(f))} \geqslant \frac{(m)}{(m)} \sigma_{f \circ g}^{L^{*}} \text { for } n>1 .
$$

Analogously from (3) and (22) and in view of the condition $\underset{(\mathfrak{p})}{(\mathfrak{m})} \rho_{\mathrm{f} \circ \mathrm{g}}^{\mathrm{L}^{*}}={ }_{(\mathfrak{p})} \rho_{\mathrm{f}}^{\mathrm{L}}$ we get that

$$
\limsup _{r \rightarrow \infty} \frac{\log ^{[m-1]} T(r, f \circ g)}{T(r, L(f))} \geqslant \frac{(m)}{\{1+k-k \delta(\infty ; f)\} \cdot(p) \sigma_{f}^{L^{*}}} .
$$

Thus the theorem follows from (20), (21), (23) and (24).

The following theorem can be carried out in the line of Theorem 3 and therefore we omit its proof.

Theorem 4 If $\mathrm{f}$ be meromorphic and $\mathrm{g}$ be transcendental entire with $0<$ ${ }_{(\mathrm{p})}^{(\mathrm{m})} \sigma_{f \circ g}^{\mathrm{L}^{*}}<\infty, 0<\underset{(\mathrm{p})}{(\mathfrak{n})} \sigma_{\mathrm{g}}^{\mathrm{L}^{*}}<\infty, \underset{(\mathrm{p})}{(\mathrm{m})} \rho_{\text {fog }}^{\mathrm{L}^{*}}={ }_{(\mathrm{p})}^{(\mathfrak{n})} \rho_{\mathrm{g}}^{\mathrm{L}^{*}}$ and $\sum_{a \neq \infty} \delta(\mathrm{a} ; \mathrm{g})+\delta(\infty ; \mathrm{g})=2$ where $\mathrm{m}, \mathrm{n}$ and $\mathrm{p}$ are any three positive integers, then

$$
\begin{aligned}
\liminf _{r \rightarrow \infty} \frac{\log ^{[m-1]} \mathrm{T}(r, f \circ g)}{\mathrm{T}(\mathrm{r}, \mathrm{L}(\mathrm{g}))} & \leq \frac{(\mathrm{m})}{\{1+\mathrm{k}-\mathrm{k} \delta(\infty ; g)\} \cdot(\mathrm{p}) \sigma_{\mathrm{f}}^{\mathrm{L}^{*}}} \\
& \leq \operatorname{limsupliminf}_{\mathrm{r} \rightarrow \infty} \frac{\log ^{[\mathrm{m}-1]} \mathrm{T}(\mathrm{r}, \mathrm{f} \circ \mathrm{g})}{\mathrm{T}(\mathrm{r}, \mathrm{L}(\mathrm{g}))}
\end{aligned}
$$

and

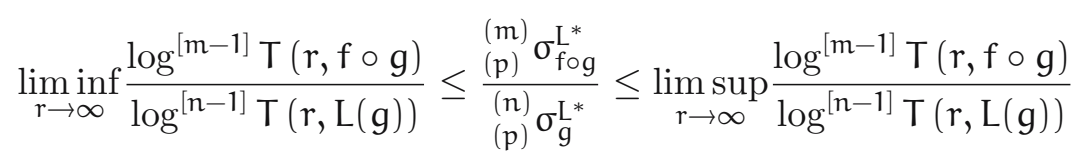

for $\mathrm{n}>1$.

The following theorem is a natural consequence of Theorem 1 and Theorem 3.

Theorem 5 If $\mathrm{f}$ be transcendental meromorphic and $\mathrm{g}$ be entire such that

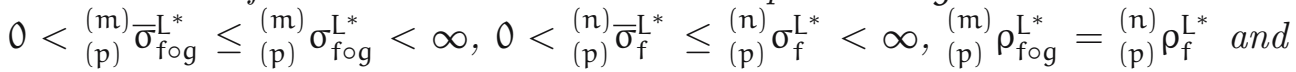
$\sum_{a \neq \infty} \delta(a ; f)+\delta(\infty ; f)=2$ where $m, n$ and $p$ are any three positive integers, 
then

$$
\begin{aligned}
\liminf _{r \rightarrow \infty} \frac{\log ^{[m-1]} T(r, f \circ g)}{T(r, L(f))} & \leq \min \left\{\frac{(p) \bar{\sigma}_{f \circ g}^{L^{*}}}{A \cdot(p)}, \frac{(p)}{\bar{\sigma}_{f}^{L^{*}}}, \frac{(m)}{A \cdot \sigma_{f(p)}^{L^{*}} \sigma_{f}^{L^{*}}}\right\} \\
& \leq \max \left\{\frac{(p) \bar{\sigma}_{f \circ g}^{L^{*}}, \frac{(p)}{(m)} \sigma_{f \circ g}^{L^{*}}}{A \cdot(p) \bar{\sigma}_{f}^{L^{*}}} \frac{(p) \sigma_{f}^{L^{*}}}{A \cdot(p)}\right\} \\
& \leq \limsup _{r \rightarrow \infty} \frac{\log ^{[m-1]} T(r, f \circ g)}{T(r, L(f))}
\end{aligned}
$$

where $A=\{1+k-k \delta(\infty ; f)\}$ and

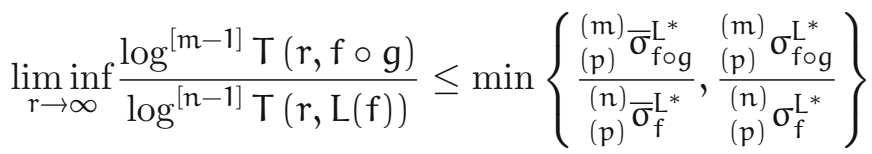

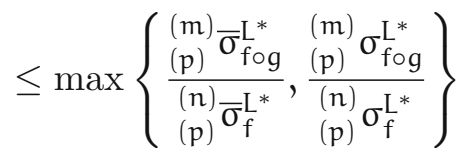

$$
\begin{aligned}
& \leq \limsup _{r \rightarrow \infty} \frac{\log ^{[m-1]} \mathrm{T}(r, f \circ g)}{\log ^{[n-1]} \mathrm{T}(r, \mathrm{~L}(\mathrm{f}))}
\end{aligned}
$$

for $n>1$.

Analogously one may state the following theorem without its proof.

Theorem 6 If $\mathrm{f}$ be meromorphic and $\mathrm{g}$ be transcendental entire with $0<$ ${ }_{(\mathrm{p})}^{(\mathrm{m})} \bar{\sigma}_{\mathrm{f} \circ \mathrm{g}}^{\mathrm{L}^{*}} \leq{ }_{(\mathrm{p})}^{(\mathrm{m})} \sigma_{\mathrm{f} \circ \mathrm{g}}^{\mathrm{L}^{*}}<\infty, 0<{ }_{(\mathrm{p})}^{(\mathrm{n})} \bar{\sigma}_{\mathrm{g}}^{\mathrm{L}^{*}} \leq{ }_{(\mathrm{p})}^{(\mathrm{n})} \sigma_{\mathrm{g}}^{\mathrm{L}^{*}}<\infty,{ }_{(\mathrm{p})}^{(\mathrm{m})} \rho_{\mathrm{f} \circ \mathrm{g}}^{\mathrm{L}^{*}}={ }_{(\mathrm{p})}^{(\mathrm{n})} \rho_{\mathrm{g}}^{\mathrm{L}^{*}}$ and $\sum_{a \neq \infty} \delta(a ; g)+\delta(\infty ; g)=2$ where $\mathrm{m}, \mathrm{n}$ and $\mathrm{p}$ are any three positive integers, then

$$
\begin{aligned}
\liminf _{r \rightarrow \infty} \frac{\log ^{[m-1]} T(r, f \circ g)}{T(r, L(g))} & \leq \min \left\{\frac{(p) \bar{\sigma}_{f \circ g}^{L^{*}}, \frac{(p)}{(m)} \sigma_{f \circ g}^{L^{*}}}{B \cdot(p) \bar{\sigma}_{g}^{L^{*}}}, \frac{(p) \sigma_{g}^{L^{*}}}{B \cdot(m)}\right\} \\
& \leq \max \left\{\frac{(p) \bar{\sigma}_{f \circ g}^{L^{*}}, \frac{(p)}{(p)} \sigma_{f \circ g}^{L^{*}}}{B \cdot(p) \bar{\sigma}_{g}^{L^{*}}, \frac{(p)}{B \cdot \sigma_{g}^{L^{*}}}}\right\} \\
& \leq \limsup _{r \rightarrow \infty} \frac{\log ^{[m-1]} T(r, f \circ g)}{T(r, L(g))}
\end{aligned}
$$


where $\mathrm{B}=\{1+\mathrm{k}-\mathrm{k} \delta(\infty ; \mathrm{g})\}$ and

$$
\begin{aligned}
& \liminf _{r \rightarrow \infty} \frac{\log ^{[m-1]} T(r, f \circ g)}{\log ^{[n-1]} T(r, L(g))} \leq \min \left\{\begin{array}{l}
(m) \bar{\sigma}_{f \circ g}^{L^{*}} \\
\frac{(m)}{(n)} \bar{\sigma}_{g}^{L^{*}}, \frac{(p)}{(n)} \sigma_{f \circ g}^{L^{*}} \\
(p) \sigma_{g}^{L^{*}}
\end{array}\right\} \\
& \leq \max \left\{\begin{array}{ll}
(m) & (p) \bar{\sigma}_{f \circ g}^{L^{*}} \\
\frac{(m)}{(n)} \bar{\sigma}_{g}^{L^{*}}, & \frac{(p)}{(n)} \sigma_{f \circ g}^{L^{*}} \\
(p) & (p) \\
\left(L_{g}^{*}\right.
\end{array}\right\} \\
& \leq \limsup _{r \rightarrow \infty} \frac{\log ^{[m-1]} \mathrm{T}(r, f \circ g)}{\log ^{[n-1]} \mathrm{T}(r, \mathrm{~L}(\mathrm{~g}))}
\end{aligned}
$$

for $n>1$.

Now in the line of Theorem 1, Theorem 3, Theorem 5 and Theorem 2, Theorem 4, Theorem 6 respectively and with the help of Lemma 2 one can easily prove the following six theorems using the notion of generalised $\mathrm{L}^{*}$-weak type with rate $p$ and therefore their proofs are omitted.

Theorem 7 If $\mathrm{f}$ be transcendental meromorphic and $\mathrm{g}$ be entire such that $0<{ }_{(p)}^{(m)} \tau_{f \circ g}^{L^{*}} \leq{ }_{(p)}^{(m)} \bar{\tau}_{f \circ g}^{L^{*}}<\infty, 0<{ }_{(p)}^{(n)} \tau_{f}^{L^{*}} \leq{ }_{(p)}^{(n)} \bar{\tau}_{f}^{L^{*}}<\infty,{ }_{(p)}^{(m)} \lambda_{f \circ g}^{L^{*}}={ }_{(p)}^{(n)} \lambda_{f}^{L^{*}}$ and $\sum_{a \neq \infty} \delta(a ; f)+\delta(\infty ; f)=2$ where $m, n$ and $p$ are any three positive integers, then

$$
\begin{aligned}
\frac{(m)}{\{1+k-k \delta(\infty ; f)\} \cdot \tau_{f \circ)}^{L^{*}} \bar{\tau}_{f}^{L^{*}}} & \leq \liminf _{r \rightarrow \infty} \frac{\log ^{[m-1]} T(r, f \circ g)}{T(r, L(f))} \\
& \leq \frac{(p) \tau_{f \circ g}^{L^{*}}}{\{1+k-k \delta(\infty ; f)\} \cdot(p) \tau_{f}^{L^{*}}} \\
& \leq \limsup _{r \rightarrow \infty} \frac{\log ^{[m-1]} T(r, f \circ g)}{T(r, L(f))} \\
& \leq \frac{(m)}{\{1+k-k \delta(\infty ; f)\} \cdot(p) \tau_{f}^{L^{*}}}
\end{aligned}
$$

and

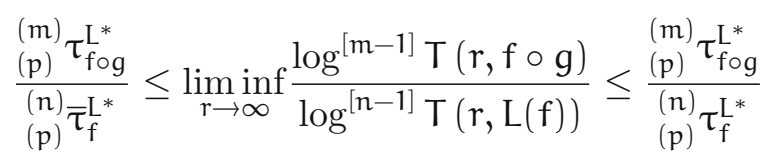




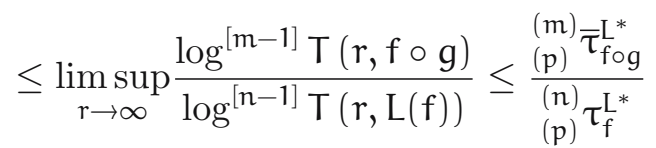

for $\mathrm{n}>1$.

Theorem 8 If $\mathrm{f}$ be transcendental meromorphic and $\mathrm{g}$ be entire with $0<$

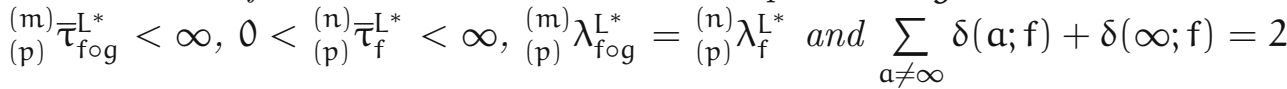
where $\mathrm{m}, \mathrm{n}$ and $\mathrm{p}$ are any three positive integers, then

$$
\begin{aligned}
\liminf _{r \rightarrow \infty} \frac{\log ^{[m-1]} T(r, f \circ g)}{T(r, L(f))} & \leq \frac{(m) \bar{\tau}_{f \circ g}^{L^{*}}}{\{1+k-k \delta(\infty ; f)\} \cdot(p) \bar{\tau}_{f}^{L^{*}}} \\
& \leq \limsup _{r \rightarrow \infty} \frac{\log ^{[m-1]} T(r, f \circ g)}{T(r, L(f))}
\end{aligned}
$$

and

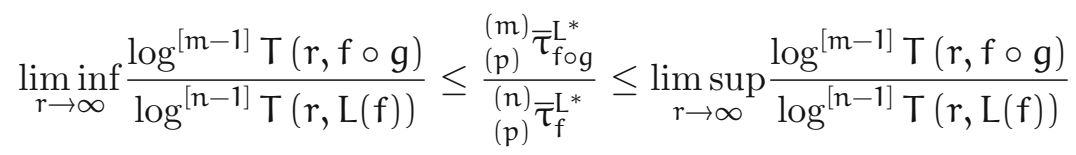

for $\mathrm{n}>1$.

Theorem 9 If $\mathrm{f}$ be transcendental meromorphic and $\mathrm{g}$ be entire such that $0<{ }_{(p)}^{(m)} \tau_{f \circ g}^{\mathrm{L}^{*}} \leq{ }_{(\mathrm{p})}^{(\mathrm{m})} \bar{\tau}_{\text {fog }}^{\mathrm{L}^{*}}<\infty, 0<{ }_{(\mathrm{p})}^{(\mathrm{n})} \tau_{\mathrm{f}}^{\mathrm{L}^{*}} \leq{ }_{(\mathrm{p})}^{(\mathrm{n})} \bar{\tau}_{\mathrm{f}}^{\mathrm{L}^{*}}<\infty,{ }_{(\mathrm{p})}^{(\mathrm{m})} \lambda_{\mathrm{fog}}^{\mathrm{L}^{*}}={ }_{(\mathrm{p})}^{(\mathrm{n})} \lambda_{\mathrm{f}}^{\mathrm{L}^{*}}$ and $\sum_{a \neq \infty} \delta(a ; f)+\delta(\infty ; f)=2$ where $\mathrm{m}, \mathrm{n}$ and $\mathrm{p}$ are any three positive integers, then

$$
\begin{aligned}
& \liminf _{r \rightarrow \infty} \frac{\log ^{[m-1]} T(r, f \circ g)}{T(r, L(f))} \leq \min \left\{\frac{(p) \tau_{f \circ g}^{(m)}, \frac{(m)}{L^{*}} \bar{\tau}_{f \circ g}^{L^{*}}}{A \cdot(p) \tau_{f}^{L^{*}}}, \frac{(p) \bar{\tau}_{f}^{L^{*}}}{A}\right\}
\end{aligned}
$$

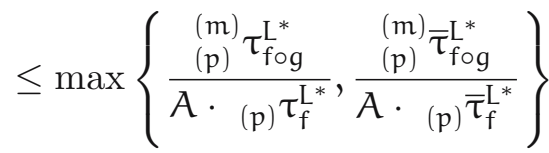

$$
\begin{aligned}
& \leq \limsup _{r \rightarrow \infty} \frac{\log ^{[m-1]} \mathrm{T}(r, f \circ g)}{T(r, L(f))}
\end{aligned}
$$


where $A=\{1+k-k \delta(\infty ; f)\}$ and

$$
\begin{aligned}
\liminf _{r \rightarrow \infty} \frac{\log ^{[m-1]} T(r, f \circ g)}{\log ^{[n-1]} T(r, L(f))} \leq \min \left\{\begin{array}{l}
(m) \tau_{f \circ g}^{L^{*}} \\
\frac{(p)}{(n)} \tau^{L^{*}}, \frac{(m)}{(n)} \bar{\tau}_{f \circ g}^{L^{*}} \\
(p) \bar{\tau}_{f}^{L^{*}}
\end{array}\right\} \\
\leq \max \left\{\begin{array}{l}
(m) \tau_{f \circ g}^{L^{*}}, \frac{(m)}{(p)} \bar{\tau}_{f \circ g}^{L^{*}} \\
\frac{(p)}{(n)} \tau_{f}^{L^{*}}, \frac{(n)}{(p)} \bar{\tau}_{f}^{L^{*}}
\end{array}\right\} \leq \limsup _{r \rightarrow \infty} \frac{\log ^{[m-1]} T(r, f \circ g)}{\log ^{[n-1]} T(r, L(f))}
\end{aligned}
$$

for $\mathrm{n}>1$.

Theorem 10 If $\mathrm{f}$ be meromorphic and $\mathrm{g}$ be transcendental entire with $0<$ ${ }_{(\mathrm{p})}^{(\mathrm{m})} \tau_{\mathrm{fog}}^{\mathrm{L}^{*}} \leq{ }_{(\mathrm{p})}^{(\mathrm{m})} \bar{\tau}_{\mathrm{fog}}^{\mathrm{L}^{*}}<\infty, 0<{ }_{(\mathrm{p})}^{(\mathrm{n})} \tau_{\mathrm{g}}^{\mathrm{L}^{*}} \leq \underset{(\mathrm{p})}{{ }_{(\mathrm{n})} \bar{\tau}_{\mathrm{g}}^{\mathrm{L}^{*}}}<\infty,{ }_{(\mathrm{p})}^{(\mathrm{m})} \lambda_{\mathrm{fog}}^{\mathrm{L}^{*}}={ }_{(\mathrm{p})}^{(\mathrm{n})} \lambda_{\mathrm{g}}^{\mathrm{L}^{*}}$ and $\sum_{\mathrm{a} \neq \infty} \delta(\mathrm{a} ; \mathrm{g})+\delta(\infty ; \mathrm{g})=2$ where $\mathrm{m}, \mathrm{n}$ and $\mathrm{p}$ are any three positive integers, then

$$
\begin{aligned}
\frac{(m)}{\{1+k-k \delta(\infty ; g)\} \cdot(p) \bar{\tau}_{g}^{L^{*}}} & \leq \liminf _{r \rightarrow \infty} \frac{\log ^{[m-1]} T(r, f \circ g)}{T(r, L(g))} \\
& \leq \frac{(p) \tau_{f \circ g}^{L^{*}}}{\{1+k-k \delta(\infty ; g)\} \cdot(p) \tau_{g}^{L^{*}}} \\
& \leq \limsup _{r \rightarrow \infty} \frac{\log ^{[m-1]} T(r, f \circ g)}{T(r, L(g))} \\
& \leq \frac{(m)}{\{1+k-k \delta(\infty ; g)\} \cdot(p) \tau_{g}^{L^{*}}}
\end{aligned}
$$

and

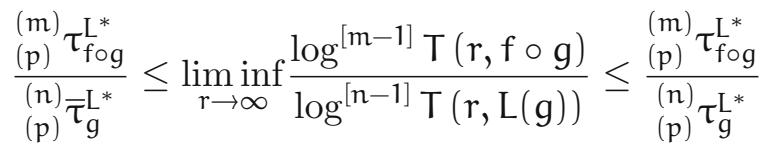

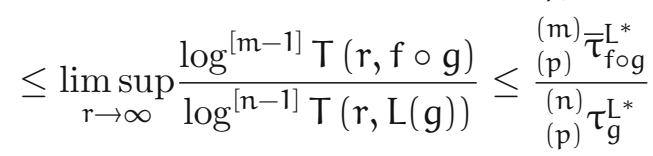

for $\mathrm{n}>1$. 
Theorem 11 If $\mathrm{f}$ be meromorphic and $\mathrm{g}$ be transcendental entire such that $0<\underset{(\mathrm{p})}{(\mathrm{m})} \bar{\tau}_{\mathrm{fog}}^{\mathrm{L}^{*}}<\infty, 0<\underset{(\mathrm{p})}{(\mathrm{n})} \bar{\tau}_{\mathrm{g}}^{\mathrm{L}^{*}}<\infty, \underset{(\mathrm{p})}{(\mathrm{m})} \lambda_{\text {fog }}^{\mathrm{L}^{*}}=\underset{(\mathrm{p})}{(\mathrm{n})} \lambda_{\mathrm{g}}^{\mathrm{L}^{*}}$ and $\sum_{\mathrm{a} \neq \infty} \delta(\mathrm{a} ; \mathrm{g})+\delta(\infty ; \mathrm{g})=$ 2 where $\mathrm{m}, \mathrm{n}$ and $\mathrm{p}$ are any three positive integers, then

$$
\begin{aligned}
\liminf _{r \rightarrow \infty} \frac{\log ^{[m-1]} \mathrm{T}(r, f \circ g)}{T(r, L(g))} & \leq \frac{(m) \bar{\tau}_{f \circ g}^{L^{*}}}{\{1+k-k \delta(\infty ; g)\} \cdot(p) \bar{\tau}_{g}^{L^{*}}} \\
& \leq \limsup _{r \rightarrow \infty} \frac{\log ^{[m-1]} \mathrm{T}(r, f \circ g)}{T(r, L(g))}
\end{aligned}
$$

and

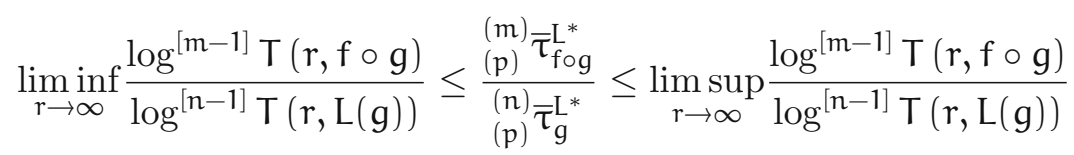

for $n>1$.

Theorem 12 If $\mathrm{f}$ be meromorphic and $\mathrm{g}$ be transcendental entire with $0<$ ${ }_{(\mathrm{p})}^{(\mathrm{m})} \tau_{\text {fog }}^{\mathrm{L}^{*}} \leq{ }_{(\mathrm{p})}^{(\mathrm{m})} \bar{\tau}_{\text {fog }}^{\mathrm{L}^{*}}<\infty, 0<{ }_{(\mathrm{p})}^{(\mathrm{n})} \tau_{\mathrm{g}}^{\mathrm{L}^{*}} \leq \underset{(\mathrm{p})}{{ }_{(\mathrm{n})}} \bar{\tau}_{\mathrm{g}}^{\mathrm{L}^{*}}<\infty, \underset{(\mathrm{p})}{(\mathrm{m})} \lambda_{\mathrm{fog}}^{\mathrm{L}^{*}}={ }_{(\mathrm{p})}^{(\mathrm{n})} \lambda_{\mathrm{g}}^{\mathrm{L}^{*}}$ and $\sum_{a \neq \infty} \delta(a ; g)+\delta(\infty ; g)=2$ where $m, n$ and $p$ are any three positive integers, then

$$
\begin{aligned}
\liminf _{r \rightarrow \infty} \frac{\log ^{[m-1]} T(r, f \circ g)}{T(r, L(g))} & \leq \min \left\{\frac{(p) \tau_{f \circ g}^{L^{*}}}{B \cdot(p) \tau_{g}^{L^{*}}}, \frac{(p)}{B \cdot(p) \bar{\tau}_{g}^{L^{*}}}\right\} \\
& \leq \max \left\{\frac{(p)}{B \cdot(p) \tau_{f \circ g}^{L^{*}}}, \frac{(p)}{B \cdot(p) \bar{\tau}_{f \circ g}^{L^{L^{*}}}}\right\} \\
& \leq \limsup _{r \rightarrow \infty} \frac{\log ^{[m-1]} T(r, f \circ g)}{T(r, L(g))}
\end{aligned}
$$

where $\mathrm{B}=\{1+\mathrm{k}-\mathrm{k} \delta(\infty ; \mathrm{g})\}$ and

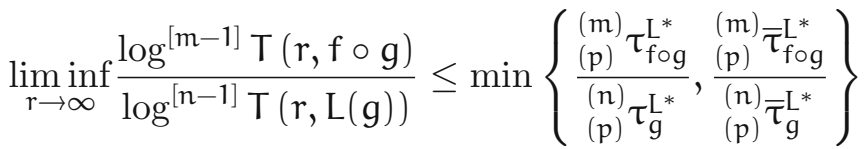

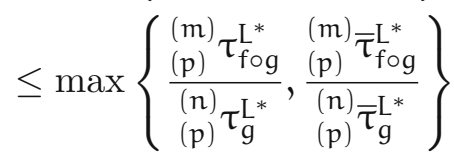

$$
\begin{aligned}
& \leq \limsup _{r \rightarrow \infty} \frac{\log ^{[m-1]} \mathrm{T}(r, f \circ g)}{\log ^{[n-1]} \mathrm{T}(r, \mathrm{~L}(\mathrm{~g}))}
\end{aligned}
$$


for $\mathrm{n}>1$.

We may now state the following theorems without their proofs based on generalised ${ }_{p} L^{*}$ - type with rate $p$ and generalised ${ }_{p} L^{*}$-weak type with rate $p$.

Theorem 13 If $\mathrm{f}$ be transcendental meromorphic and $\mathrm{g}$ be entire such that $0<\underset{(p)}{(m)} \bar{\sigma}_{f \circ g}^{L^{*}} \leq{ }_{(p)}^{(m)} \sigma_{f \circ g}^{L^{*}}<\infty, 0<\underset{(p)}{(n)} \tau_{f}^{L^{*}} \leq \underset{(p)}{(n)} \bar{\tau}_{f}^{L^{*}}<\infty, \underset{(p)}{(m)} \rho_{f \circ g}^{L^{*}}={ }_{(p)}^{(n)} \lambda_{f}^{L^{*}}$ and $\sum_{a \neq \infty} \delta(a ; f)+\delta(\infty ; f)=2$ where $m, n$ and $p$ are any three positive integers, then

$$
\begin{aligned}
\frac{(m)}{\{1+k-k \delta(\infty ; f)\} \cdot(p) \bar{\tau}_{f}^{L_{f}^{*}}} & \leq \liminf _{r \rightarrow \infty} \frac{\log ^{[m-1]} T(r, f \circ g)}{T(r, L(f))} \\
& \leq \frac{(m){ }_{f \circ g}^{L^{*}}}{\{1+k-k \delta(\infty ; f)\} \cdot(p) \tau_{f}^{L^{*}}} \\
& \leq \limsup _{r \rightarrow \infty} \frac{\log ^{[m-1]} T(r, f \circ g)}{T(r, L(f))} \\
& \leq \frac{(m)}{\{1+k-k \delta(\infty ; f)\} \cdot(p) \tau_{f}^{L^{*}}}
\end{aligned}
$$

and

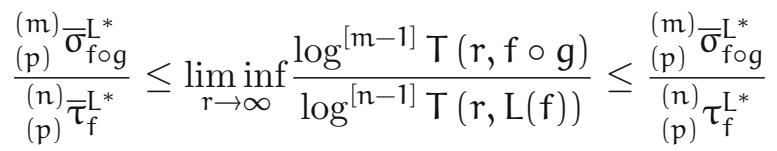

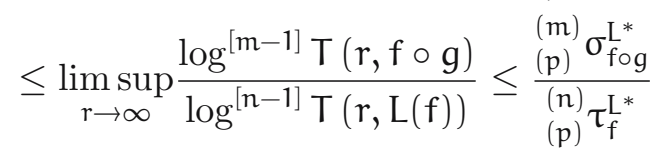

for $\mathrm{n}>1$.

Theorem 14 If $\mathrm{f}$ be transcendental meromorphic and $\mathrm{g}$ be entire with $0<$ ${ }_{(\mathrm{p})}^{(m)} \sigma_{f \circ g}^{\mathrm{L}^{*}}<\infty, 0<\underset{(\mathrm{p})}{(\mathrm{n})} \bar{\tau}_{\mathrm{f}}^{\mathrm{L}^{*}}<\infty, \underset{(\mathrm{p})}{(\mathrm{m})} \rho_{\text {fog }}^{\mathrm{L}^{*}}=\underset{(\mathrm{p})}{(\mathrm{n})} \lambda_{\mathrm{f}}^{\mathrm{L}^{*}}$ and $\sum_{\mathrm{a} \neq \infty} \delta(\mathrm{a} ; \mathrm{f})+\delta(\infty ; f)=2$ where $\mathrm{m}, \mathrm{n}$ and $\mathrm{p}$ are any three positive integers, then

$$
\begin{aligned}
\liminf _{r \rightarrow \infty} \frac{\log ^{[m-1]} T(r, f \circ g)}{T(r, L(f))} & \leq \frac{(m)}{(p) \sigma_{f \circ g}^{L^{*}}} \\
& \leq \limsup _{r \rightarrow \infty} \frac{\log ^{[m-1]} T(r, f \circ g)}{T(r, L(f))}
\end{aligned}
$$


and

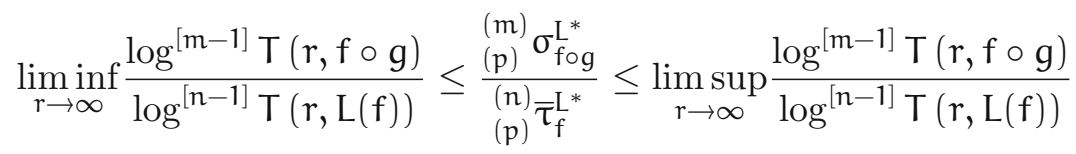

for $\mathrm{n}>1$.

Theorem 15 Let $\mathrm{f}$ be transcendental meromorphic and $\mathrm{g}$ be entire such that $0<\underset{(p)}{(m)} \bar{\sigma}_{f \circ g}^{L^{*}} \leq{ }_{(p)}^{(m)} \sigma_{f \circ g}^{L^{*}}<\infty, 0<{ }_{(p)}^{(n)} \tau_{f}^{L^{*}} \leq{ }_{(p)}^{(n)} \bar{\tau}_{f}^{L^{*}}<\infty, \underset{(p)}{(m)} \rho_{f \circ g}^{L^{*}}={ }_{(p)}^{(n)} \lambda_{f}^{L^{*}}$ and $\sum_{a \neq \infty} \delta(a ; f)+\delta(\infty ; f)=2$ where $m, n$ and $p$ are any three positive integers, then

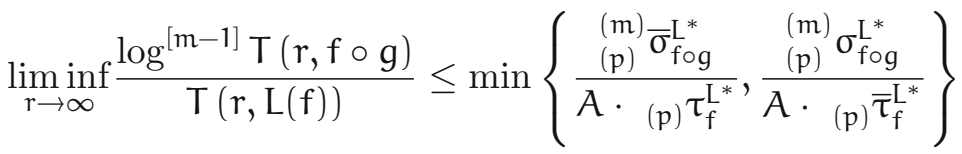

$$
\begin{aligned}
& \leq \max \left\{\frac{(\mathfrak{m}) \bar{\sigma}_{f \circ g}^{L^{*}}}{A \cdot(p) \tau_{f}^{L^{*}}}, \frac{(p)}{A \cdot(p) \sigma_{f \circ g}^{L_{f}^{*}}}\right\} \\
& \leq \limsup _{r \rightarrow \infty} \frac{\log ^{[m-1]} \mathrm{T}(r, f \circ g)}{\mathrm{T}(r, \mathrm{~L}(\mathrm{f}))}
\end{aligned}
$$

where $A=\{1+k-k \delta(\infty ; f)\}$ and

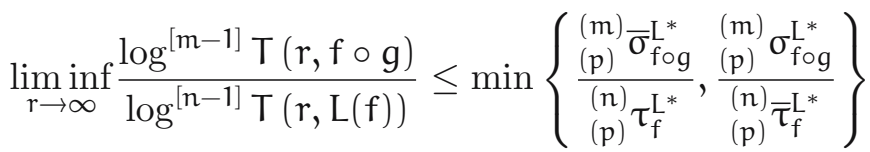

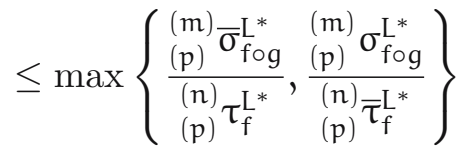

$$
\begin{aligned}
& \leq \limsup _{r \rightarrow \infty} \frac{\log ^{[m-1]} \mathrm{T}(r, f \circ g)}{\log ^{[n-1]} \mathrm{T}(r, \mathrm{~L}(\mathrm{f}))}
\end{aligned}
$$

for $n>1$.

Theorem 16 If $\mathrm{f}$ be transcendental meromorphic and $\mathrm{g}$ be entire with $0<$

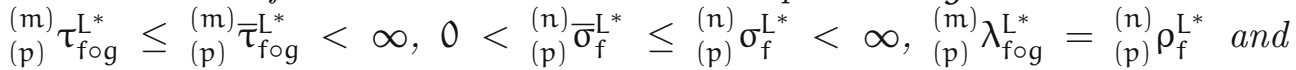
$\sum_{a \neq \infty} \delta(a ; f)+\delta(\infty ; f)=2$ where $m, n$ and $p$ are any three positive integers, 
then

$$
\begin{aligned}
\frac{(m)}{\{1+k-k \delta(\infty ; f)\} \cdot \tau_{f \circ}^{L^{*}}} \sigma_{f}^{L^{*}} & \leq \liminf _{r \rightarrow \infty} \frac{\log ^{[m-1]} T(r, f \circ g)}{T(r, L(f))} \\
& \leq \frac{(p) \tau_{f \circ g}^{L^{*}}}{\{1+k-k \delta(\infty ; f)\} \cdot(p) \bar{\sigma}_{f}^{L^{*}}} \\
& \leq \limsup _{r \rightarrow \infty} \frac{\log ^{[m-1]} T(r, f \circ g)}{T(r, L(f))} \\
& \leq \frac{(m) \tau_{f \circ g}^{L^{*}}}{\{1+k-k \delta(\infty ; f)\} \cdot(p) \bar{\sigma}_{f}^{L^{*}}}
\end{aligned}
$$

and

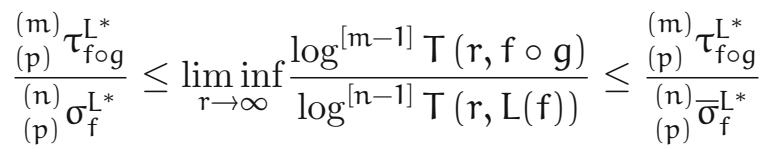

$$
\begin{aligned}
& \leq \limsup _{r \rightarrow \infty} \frac{\log ^{[m-1]} \mathrm{T}(r, f \circ g)}{\log ^{[n-1]} \mathrm{T}(r, L(f))} \leq \frac{(\mathrm{m}) \bar{\tau}_{f \circ g}^{L^{*}}}{(\mathfrak{n}) \bar{\sigma}_{f}^{L^{*}}}
\end{aligned}
$$

for $\mathrm{n}>1$.

Theorem 17 If $\mathrm{f}$ be transcendental meromorphic and $\mathrm{g}$ be entire such that $0<\underset{(p)}{(m)} \bar{\tau}_{f \circ g}^{L^{*}}<\infty, 0<\underset{(p)}{(n)} \sigma_{f}^{L^{*}}<\infty, \underset{(p)}{(m)} \lambda_{f \circ g}^{L^{*}}=\underset{(p)}{(n)} \rho_{f}^{L^{*}}$ and $\sum_{a \neq \infty} \delta(a ; f)+\delta(\infty ; f)=$ 2 where $\mathrm{m}, \mathrm{n}$ and $\mathrm{p}$ are any three positive integers, then

$$
\begin{aligned}
\liminf _{r \rightarrow \infty} \frac{\log ^{[m-1]} T(r, f \circ g)}{T(r, L(f))} & \leq \frac{(m) \bar{\tau}_{f \circ g}^{L^{*}}}{\{1+k-k \delta(\infty ; f)\} \cdot(p) \sigma_{f}^{L^{*}}} \\
& \leq \limsup _{r \rightarrow \infty} \frac{\log ^{[m-1]} T(r, f \circ g)}{T(r, L(f))}
\end{aligned}
$$

and

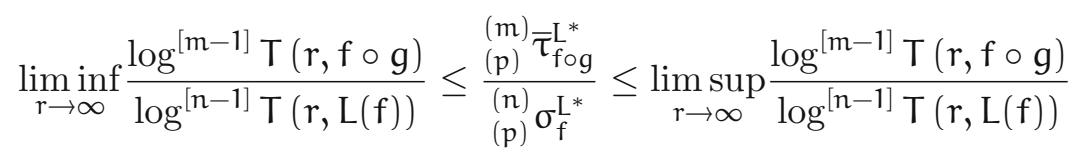

for $n>1$. 
Theorem 18 If $\mathrm{f}$ be transcendental meromorphic and $\mathrm{g}$ be entire with $0<$ ${ }_{(\mathrm{p})}^{(\mathrm{m})} \tau_{\mathrm{fog}}^{\mathrm{L}^{*}} \leq \underset{(\mathrm{p})}{{ }_{(\mathrm{m})} \bar{\tau}_{\text {fog }}^{\mathrm{L}^{*}}}<\infty, 0<{ }_{(\mathrm{p})}^{(\mathrm{n})} \bar{\sigma}_{\mathrm{f}}^{\mathrm{L}^{*}} \leq{ }_{(\mathrm{p})}^{(\mathrm{n})} \sigma_{\mathrm{f}}^{\mathrm{L}^{*}}<\infty, \underset{(\mathrm{p})}{(\mathrm{m})} \lambda_{\text {fog }}^{\mathrm{L}^{*}}={ }_{(\mathrm{p})}^{(\mathrm{n})} \rho_{\mathrm{f}}^{\mathrm{L}^{*}}$ and $\sum_{a \neq \infty} \delta(a ; f)+\delta(\infty ; f)=2$ where $m, n$ and $p$ are any three positive integers, then

$$
\begin{aligned}
\liminf _{r \rightarrow \infty} \frac{\log ^{[m-1]} T(r, f \circ g)}{T(r, L(f))} & \leq \min \left\{\frac{(m) \tau_{f \circ g}^{L^{*}}}{A \cdot(p) \bar{\sigma}_{f}^{L^{*}}}, \frac{(p)}{A \cdot(p) \sigma_{f o g}^{L^{*}}}\right\} \\
& \leq \max \left\{\frac{(p) \tau_{f \circ g}^{L^{*}}}{A \cdot(p) \bar{\sigma}_{f}^{L^{*}}, \frac{(p)}{A \cdot(p)} \bar{\tau}_{f \circ g}^{L_{f}} \sigma_{f}^{L^{*}}}\right\} \\
& \leq \limsup _{r \rightarrow \infty} \frac{\log ^{[m-1]} T(r, f \circ g)}{T(r, L(f))}
\end{aligned}
$$

where $A=\{1+k-k \delta(\infty ; f)\}$ and

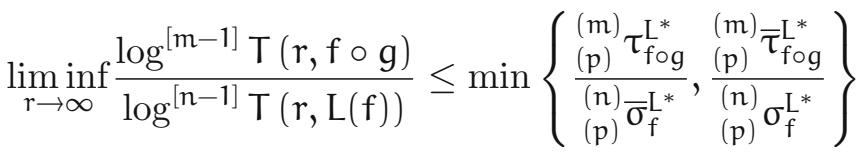

$$
\begin{aligned}
& \leq \max \left\{\begin{array}{ll}
(m) & (p) \tau_{f \circ g}^{L^{*}} \\
\frac{(m)}{(n)} \bar{\tau}_{f \circ g}^{L^{*}} & \frac{(p)}{(n)} \bar{\sigma}_{f}^{L^{*}} \\
(p) & (p)
\end{array}\right\} \\
& \leq \limsup _{r \rightarrow \infty} \frac{\log ^{[m-1]} \mathrm{T}(r, f \circ g)}{\log ^{[n-1]} \mathrm{T}(r, \mathrm{~L}(\mathrm{f}))}
\end{aligned}
$$

for $\mathrm{n}>1$.

Theorem 19 If $\mathrm{f}$ be meromorphic and $\mathrm{g}$ be transcendental entire such that $0<\underset{(p)}{(m)} \bar{\sigma}_{f \circ g}^{L^{*}} \leq{ }_{(p)}^{(m)} \sigma_{f \circ g}^{L^{*}}<\infty, 0<\underset{(p)}{(n)} \tau_{g}^{L^{*}} \leq \underset{(p)}{(n)} \bar{\tau}_{g}^{L^{*}}<\infty, \underset{(p)}{(m)} \rho_{f \circ g}^{L^{*}}=\underset{(p)}{(n)} \lambda_{g}^{L^{*}}$ and $\sum_{a \neq \infty} \delta(a ; g)+\delta(\infty ; g)=2$ where $m, n$ and $p$ are any three positive integers, then

$$
\begin{aligned}
\frac{(m)}{\{1+k-k \delta(\infty ; g)\} \cdot(p) \bar{\sigma}_{f \circ g}^{L^{*}}} & \leq \liminf _{r \rightarrow \infty} \frac{\log ^{[m-1]} T(r, f \circ g)}{T(r, L(g))} \\
& \leq \frac{(p){ }_{f \circ g}^{L^{2 *}}}{\{1+k-k \delta(\infty ; g)\} \cdot(p) \tau_{g}^{L^{*}}} \\
& \leq \limsup _{r \rightarrow \infty} \frac{\log ^{[m-1]} T(r, f \circ g)}{T(r, L(g))}
\end{aligned}
$$




$$
\leq \frac{(m)}{\{1+k-k \delta(\infty ; g)\} \cdot(p) \tau_{g}^{L^{*}}}
$$

and

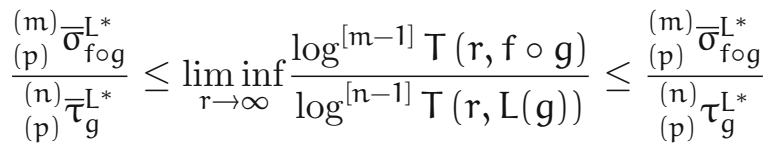

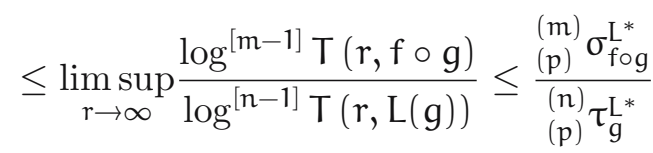

for $n>1$.

Theorem 20 If $\mathrm{f}$ be meromorphic and $\mathrm{g}$ be transcendental entire with $0<$ $\underset{(\mathrm{p})}{(\mathrm{m})} \sigma_{f \circ g}^{\mathrm{L}^{*}}<\infty, 0<\underset{(\mathrm{p})}{(\mathrm{n})} \bar{\tau}_{\mathrm{g}}^{\mathrm{L}^{*}}<\infty, \underset{(\mathrm{p})}{(\mathrm{m})} \rho_{\mathrm{f} \circ \mathrm{g}}^{\mathrm{L}^{*}}=\underset{(\mathrm{p})}{(\mathfrak{n})} \lambda_{\mathrm{g}}^{\mathrm{L}^{*}}$ and $\sum_{\mathrm{a} \neq \infty} \delta(\mathrm{a} ; \mathrm{g})+\delta(\infty ; \mathrm{g})=2$ where $\mathrm{m}, \mathrm{n}$ and $\mathrm{p}$ are any three positive integers, then

$$
\begin{aligned}
\liminf _{r \rightarrow \infty} \frac{\log ^{[m-1]} T(r, f \circ g)}{T(r, L(g))} & \leq \frac{(m)}{\{1+k-k \delta(\infty ; g)\} \cdot(p) \sigma_{f \circ g}^{L^{L^{*}}}} \\
& \leq \operatorname{limsupliminf}_{r \rightarrow \infty} \frac{\log _{r \rightarrow \infty}^{[m-1]} T(r, f \circ g)}{T(r, L(g))}
\end{aligned}
$$

and

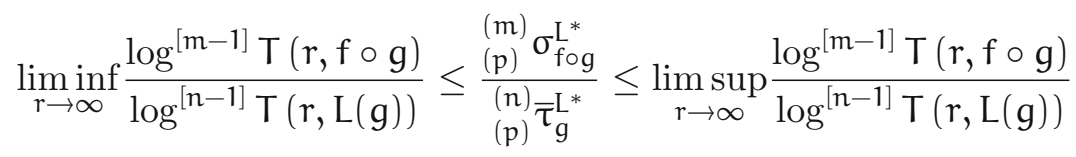

for $\mathrm{n}>1$.

Theorem 21 If $\mathrm{f}$ be meromorphic and $\mathrm{g}$ be transcendental entire such that $0<\underset{(p)}{(m)} \bar{\sigma}_{f \circ g}^{L^{*}} \leq{ }_{(p)}^{(m)} \sigma_{f \circ g}^{L^{*}}<\infty, 0<\underset{(p)}{(n)} \tau_{g}^{L^{*}} \leq \underset{(p)}{{ }_{(n)}^{(n)}} \bar{\tau}_{g}^{L^{*}}<\infty, \underset{(p)}{(m)} \rho_{f \circ g}^{L^{*}}={ }_{(p)}^{(n)} \lambda_{g}^{L^{*}}$ and $\sum_{a \neq \infty} \delta(a ; g)+\delta(\infty ; g)=2$ where $m, n$ and $p$ are any three positive integers, 
then

$$
\begin{aligned}
\liminf _{r \rightarrow \infty} \frac{\log ^{[m-1]} T(r, f \circ g)}{T(r, L(g))} & \leq \min \left\{\frac{(p) \sigma_{f \circ g}^{L^{*}}}{B \cdot(p) \tau_{g}^{L^{*}}}, \frac{(p) \sigma_{f \circ g}^{L^{*}}}{B \cdot(p) \bar{\tau}_{g}^{L^{*}}}\right\} \\
& \leq \max \left\{\frac{(p){ }_{f \circ g}^{L^{*}}}{B \cdot(p) \tau_{g}^{L^{*}}, \frac{(m)}{B \cdot(p)} \sigma_{f \circ g}^{L^{*}} \bar{\tau}_{g}^{L^{*}}}\right\} \\
& \leq \limsup _{r \rightarrow \infty} \frac{\log ^{[m-1]} T(r, f \circ g)}{T(r, L(g))}
\end{aligned}
$$

where $\mathrm{B}=\{1+\mathrm{k}-\mathrm{k} \delta(\infty ; \mathrm{g})\}$ and

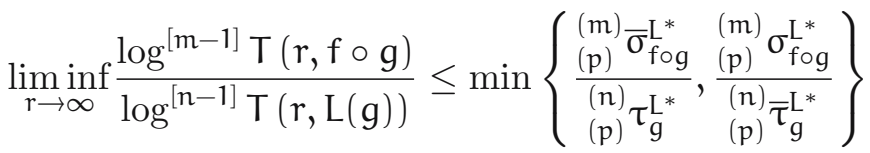

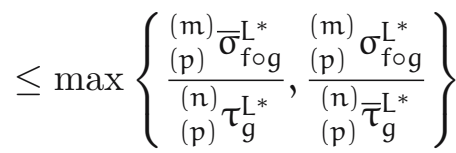

$$
\begin{aligned}
& \leq \limsup _{r \rightarrow \infty} \frac{\log ^{[m-1]} \mathrm{T}(r, f \circ g)}{\log ^{[n-1]} \mathrm{T}(r, \mathrm{~L}(\mathrm{~g}))}
\end{aligned}
$$

for $\mathrm{n}>1$.

Theorem 22 If $\mathrm{f}$ be meromorphic and $\mathrm{g}$ be transcendental entire with $0<$ ${ }_{(\mathrm{p})}^{(\mathrm{m})} \tau_{\mathrm{fog}}^{\mathrm{L}^{*}} \leq \underset{(\mathrm{p})}{{ }_{(\mathrm{m})} \bar{\tau}_{\text {fog }}^{\mathrm{L}^{*}}}<\infty, 0<\underset{(\mathrm{p})}{(\mathrm{n})} \bar{\sigma}_{\mathrm{g}}^{\mathrm{L}^{*}} \leq \underset{(\mathrm{p})}{{ }_{(\mathrm{n})}} \sigma_{\mathrm{g}}^{\mathrm{L}^{*}}<\infty, \underset{(\mathrm{p})}{(\mathrm{m})} \lambda_{\text {fog }}^{\mathrm{L}^{*}}={ }_{(\mathrm{p})}^{(\mathrm{n})} \rho_{\mathrm{g}}^{\mathrm{L}^{*}}$ and $\sum_{a \neq \infty} \delta(a ; g)+\delta(\infty ; g)=2$ where $m, n$ and $p$ are any three positive integers, then

$$
\begin{aligned}
\frac{(m)}{(m)} \tau_{f \circ g}^{L^{*}} & \leq \liminf _{r \rightarrow \infty} \frac{\log ^{[m-1]} T(r, f \circ g)}{T(r, L(g))} \\
& \leq \frac{(p) \tau_{f \circ g}^{L^{*}}}{\{1+k-k \delta(\infty ; g)\} \cdot(p) \bar{\sigma}_{g}^{L^{*}}} \\
& \leq \limsup _{r \rightarrow \infty} \frac{\log ^{[m-1]} \mathrm{T}(r, f \circ g)}{T(r, L(g))} \\
& \leq \frac{(m) \sigma_{g}^{L^{*}}}{\{1+k-k \delta(\infty ; g)\} \cdot(p) \bar{\sigma}_{g}^{L^{*}}}
\end{aligned}
$$


and

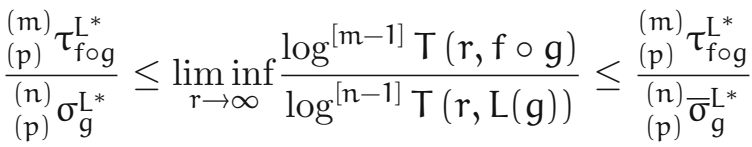

$$
\begin{aligned}
& \leq \limsup _{r \rightarrow \infty} \frac{\log ^{[m-1]} \mathrm{T}(r, f \circ g)}{\log ^{[n-1]} \mathrm{T}(r, L(g))} \leq \frac{(\mathrm{m})}{\bar{\tau}_{f \circ g}^{L^{*}}}
\end{aligned}
$$

for $\mathrm{n}>1$.

Theorem 23 If $\mathrm{f}$ be meromorphic and $\mathrm{g}$ be transcendental entire such that $0<\underset{(p)}{(m)} \bar{\tau}_{f \circ g}^{L^{*}}<\infty, 0<\underset{(p)}{(\mathfrak{n})} \sigma_{g}^{L^{*}}<\infty, \underset{(p)}{(\mathfrak{m})} \lambda_{f \circ g}^{L^{*}}=\underset{(p)}{(\mathfrak{n})} \rho_{g}^{L^{*}}$ and $\sum_{a \neq \infty} \delta(a ; g)+\delta(\infty ; g)=$ 2 where $\mathrm{m}, \mathrm{n}$ and $\mathrm{p}$ are any three positive integers, then

$$
\begin{aligned}
\liminf _{r \rightarrow \infty} \frac{\log ^{[m-1]} T(r, f \circ g)}{T(r, L(g))} & \leq \frac{(m){ }_{\tau}^{L_{f \circ g}^{*}}}{\{1+k-k \delta(\infty ; g)\} \cdot(p) \sigma_{g}^{L^{*}}} \\
& \leq \limsup _{r \rightarrow \infty} \frac{\log ^{[m-1]} T(r, f \circ g)}{T(r, L(g))}
\end{aligned}
$$

and

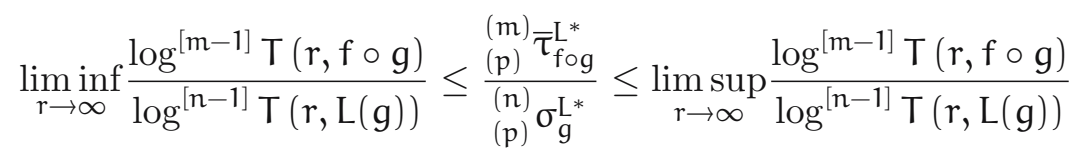

for $\mathrm{n}>1$.

Theorem 24 If $\mathrm{f}$ be transcendental meromorphic and $\mathrm{g}$ be entire with $0<$ ${ }_{(\mathrm{p})}^{(\mathrm{m})} \tau_{f \circ g}^{\mathrm{L}^{*}} \leq{ }_{(\mathrm{p})}^{(\mathrm{m})} \bar{\tau}_{\mathrm{fog}}^{\mathrm{L}^{*}}<\infty, 0<{ }_{(\mathrm{p})}^{(\mathrm{n})} \bar{\sigma}_{g}^{\mathrm{L}^{*}} \leq{ }_{(\mathrm{p})}^{(\mathfrak{n})} \sigma_{g}^{\mathrm{L}^{*}}<\infty, \underset{(\mathrm{p})}{(\mathrm{m})} \lambda_{f \circ g}^{\mathrm{L}^{*}}={ }_{(\mathrm{p})}^{(\mathfrak{n})} \rho_{g}^{\mathrm{L}^{*}}$ and $\mathrm{g}$ has the maximum deficiency sum where $\mathrm{m}, \mathrm{n}$ and $\mathrm{p}$ are any three positive integers, then

$$
\begin{aligned}
& \liminf _{r \rightarrow \infty} \frac{\log ^{[m-1]} T(r, f \circ g)}{T(r, L(g))} \leq \min \left\{\frac{(p) \tau_{f \circ g}^{(m)}, \frac{(p)}{L^{*}} \bar{\tau}_{f \circ g}^{L^{*}}}{B \cdot(p) \bar{\sigma}_{g}^{L^{*}}}, \frac{(p)}{B \cdot \sigma_{g}^{L^{*}}}\right\}
\end{aligned}
$$

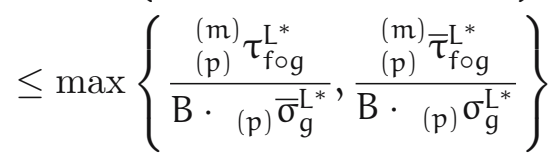

$$
\begin{aligned}
& \leq \limsup _{r \rightarrow \infty} \frac{\log ^{[m-1]} \mathrm{T}(r, f \circ g)}{\mathrm{T}(r, \mathrm{~L}(\mathrm{~g}))}
\end{aligned}
$$


where $\mathrm{B}=\{1+\mathrm{k}-\mathrm{k} \delta(\infty ; \mathrm{g})\}$ and

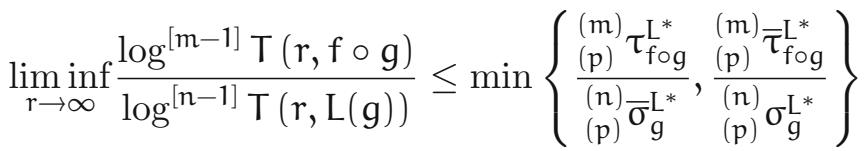

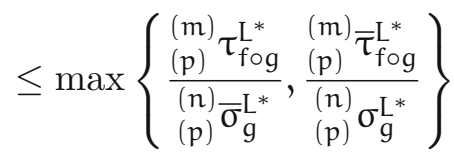

$$
\begin{aligned}
& \leq \limsup _{r \rightarrow \infty} \frac{\log ^{[m-1]} \mathrm{T}(r, f \circ g)}{\log ^{[n-1]} \mathrm{T}(\mathrm{r}, \mathrm{L}(\mathrm{g}))}
\end{aligned}
$$

for $\mathrm{n}>1$.

\section{References}

[1] S. K. Datta, A. Biswas, Some generalized growth properties of composite entire functions involving their maximum terms on the basis of slowly changing functions, Int. J. Math. Anal. (Ruse), 5 (22) (2011), 1093-1101.

[2] S. K. Datta, T. Biswas, S. Ali, Some results on wronskians using slowly changing functions, News Bull. Calcutta Math. Soc., 36 (7-9) (2013), 8-24.

[3] S. K. Datta, T. Biswas, A. Kar, On the growth analysis of wronskians in the light of some generalized growth indicators, Commun. Fac. Sci. Univ. Ank. Series A1, 64 (2) (2015), 1-34.

[4] W. K. Hayman, Meromorphic Functions, The Clarendon Press, Oxford, 1964.

[5] T. V. Lakshminarasimhan, A note on entire functions of bounded index, J. Indian Math. Soc., 38 (1974), 43-49.

[6] I. Lahiri, N. R. Bhattacharjee, Functions of L-bounded index and of nonuniform L-bounded index, Indian J. Math., 40 (1) (1998), 43-57.

[7] S. K. Singh, G. P. Barker, Slowly changing functions and their applications, Indian J. Math., 19 (1) (1977), 1-6. 
[8] D. Somasundaram, R. Thamizharasi, A note on the entire functions of L-bounded index and L-type, Indian J. Pure Appl. Math., 19 (3) (March 1988), 284-293.

[9] G. Valiron, Lectures on the general theory of integral functions, Chelsea Publishing Company, 1949.

Received: September 17, 2014 